AAS 13-337

\title{
LINEAR STATE REPRESENTATIONS FOR IDENTIFICATION OF BILINEAR DISCRETE-TIME MODELS BY INTERACTION MATRICES
}

\author{
Francesco Vicario, ${ }^{*}$ Minh Q. Phan, ${ }^{\dagger}$ \\ Raimondo Betti ${ }^{\ddagger}$ and Richard W. Longman ${ }^{\S}$
}

\begin{abstract}
Bilinear systems can be viewed as a bridge between linear and nonlinear systems, providing a promising approach to handle various nonlinear identification and control problems. This paper provides a formal justification for the extension of interaction matrices to bilinear systems and uses them to express the bilinear state as a linear function of input-output data. Multiple representations of this kind are derived, making it possible to develop an intersection subspace algorithm for the identification of discrete-time bilinear models. The technique first recovers the bilinear state by intersecting two vector spaces that are defined solely in terms of input-output data. The new input-output-to-state relationships are also used to extend the Equivalent Linear Model method for bilinear system identification. Among the benefits of the proposed approach, it does not require data from multiple experiments, and it does not impose specific restrictions on the form of input excitation.
\end{abstract}

\section{INTRODUCTION}

System identification as a research topic has attracted a lot of interest over the last decades with applications in many fields including aerospace engineering. Although well-established techniques exist for linear systems, this is not the case for nonlinear systems. Some dynamical systems are inherently bilinear such as those that involve the regulation of thermal energy by fluid flow. More importantly, by increasing the state dimension a bilinear model can be used to approximate a more general nonlinear system (Refs. [1,2,3]). Interest in bilinear systems has recently grown after a technique formally known as Carleman linearization was found to achieve such an approximation (Refs. [4,5,6]). Bilinear systems can mean systems having multiple variables, and having the property that they are linear in each variable if the remaining variables are held constant. In satellite attitude dynamics, the Euler equations for the satellite angular velocity as a function of applied torque are bilinear equations in this sense. One needs to add to these equations, additional equations that represent the kinematics of rigid body motion, which then give the satellite attitude as a function of time that results from the angular velocity history. There are various choices for these kinematic equations: one can use 9 direction cosine matrix elements, or 6 direction cosine matrix elements, or 4 parameter representations such as quaternions, Rodriguez parameters, and Euler Rodriguez parameters, or 3 parameter sets of Euler angles. The first few of these choices also produce bilinear equations in this sense, so that the satellite attitude control problem can be thought of as a set of 12 or 9 bilinear equations. The literature on bilinear systems usually makes use of a different form of bilinear equation that only contains nonlinear terms that are products of a state variable with the input. Carleman bilinearization will convert the satellite attitude dynamics bilinear problem into this form of bilinear equations (Refs. [3,7]), so that one can think of system identification of bilinear systems, developing state estimators for bilinear systems, and control methods for bilinear systems as an approach to handle the satellite attitude control problem.

*Doctoral Student, Department of Mechanical Engineering, Columbia University, New York, NY 10027, USA

${ }^{\dagger}$ Associate Professor, Thayer School of Engineering, Dartmouth College, Hanover, NH 03755, USA.

${ }_{\ddagger}^{\ddagger}$ Professor, Department of Civil Engineering and Engineering Mechanics, Columbia University, New York, NY 10027, USA.

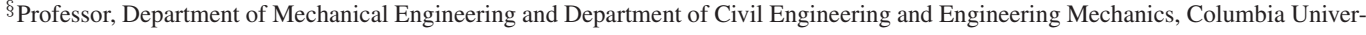
sity, New York, NY 10027, USA 
Recently a bilinear model identification method was presented (Ref. [8]), by extending the interaction matrix formulation that was originally developed for linear state-space models. A linear relationship between the bilinear system state and past input-output data was obtained and used to rewrite the bilinear model as an Equivalent Linear Model (ELM). The ELM can be identified with any linear identification method, from which the original bilinear model is recovered. In contrast to other attempts to transform the bilinear model into an ELM, e.g. by expanding the bilinear system state with sinusoidal basis functions by perturbation theory (Ref. [9]), or to apply subspace methods (Ref. [10]), a benefit of the method in Ref. [8] is the freedom in choosing the input excitation as long as it is sufficiently rich. We focus on discrete-time methods that are particularly suitable for digital implementation although physical dynamical systems are in continuous time. A first significant step towards this objective has been made in Ref. [7] that shows how a continuous-time bilinear state-space model can be approximated by a discrete-time version without losing the simple bilinear structure of the model. Direct identification of continuous-time bilinear models for a continuous-time bilinear system is another track of research that is being vigorously pursued (Refs. [11,12]).

The first fundamental contribution of the present paper is a formal proof for the existence of the interaction matrices that were originally postulated in Refs. [8,13]. These interaction matrices were used to linearly express the causal relationship between the state of a bilinear system at the current time step in terms of past input-output measurements. The second contribution is showing that the bilinear state can also be linearly related to future and current input-output, again via the interaction matrices. The derivation of the anticausal state representation is less straightforward than the causal version established in Ref. [8], but makes it possible to develop an Intersection Subspace (IS) approach to discrete-time bilinear system identification. The third contribution of this paper is therefore the IS approach where the bilinear system state history is reconstructed by intersecting two input-output vector spaces. The bilinear state-space model matrices can then be identified by the classic least-squares method. To our knowledge, this is the first application to bilinear systems of an algorithm based on intersection of vector spaces, which has proven to be very effective in linear system identification (Ref. $[14,15])$. Note that in the proposed IS method it is not even necessary to identify an ELM or explicitly compute the interaction matrices. Additionally, establishing that an anticausal linear relation also exists between the bilinear state and the input-output data gives rise to an anticausal version of the ELM-based method presented in Ref. [8]. This new development serves as a useful complement to the original method. Finally, other linear input-output state representations are also developed and their benefits are discussed, resulting in a wide range of algorithms for the identification of discrete-time bilinear systems that require data from a single experiment without specific restrictions on the form of excitation input that must be used.

\section{PROBLEM STATEMENT}

Consider an $n$-state, single-input, $q$-output bilinear system in state-space form

$$
\begin{aligned}
x(k+1) & =A x(k)+N x(k) u(k)+B u(k) \\
y(k) & =C x(k)+D u(k)
\end{aligned}
$$

A single set of length $l$ of input-output data that starts from some unknown initial state $x(0)$ is given

$$
\begin{aligned}
& \{u(k)\}=\{u(0), \quad u(1), \quad u(2), \ldots, \quad u(l-1)\} \\
& \{y(k)\}=\{y(0), \quad y(1), \quad y(2), \ldots, \quad y(l-1)\}
\end{aligned}
$$

The objective is to identify the system of Eq. (1) with the input-output data provided in Eq. (2). The data is assumed to be of sufficient length and richness so that the system of Eq. (1) can be correctly identified. For simplicity, we focus on the single-input case in this paper. Extension to the multi-input case can be made without conceptual difficulties.

\section{APPROACHES TO BILINEAR SYSTEM IDENTIFICATION}

Two different approaches to bilinear system identification are proposed in this paper. Both of them are based on the following linear relationship between the state $x(k)$ of the bilinear system and a superstate $z(k)$ 
made of input-output data only

$$
x(k)=T z(k)
$$

where $T$ is a constant matrix. In this paper, relations of the form of Eq. (3) play a central role and are referred to as Input-Output-to-State Representations (IOSRs). Depending on the specific choice of IOSR or, equivalently, on the specific definition chosen for the superstate $z(k)$, several identification algorithms of ELM and IS type can be devised.

\section{Equivalent Linear Model (ELM) Method}

If a relation like Eq. (3) was available for bilinear systems, the identification problem could be dramatically simplified by rewriting the original bilinear system of Eq. (1) in the form of a linear system. Substituting Eq. (3) into Eq. (1a) we obtain the following Equivalent Linear Model (ELM)

$$
\begin{aligned}
x(k+1) & =A x(k)+B_{E L M} w(k) \\
y(k) & =C x(k)+D u(k)
\end{aligned}
$$

where

$$
B_{E L M}=\left[\begin{array}{ll}
B & N T
\end{array}\right]
$$

and $w(k)$ is defined as the input to the ELM

$$
w(k)=\left[\begin{array}{c}
u(k) \\
z(k) u(k)
\end{array}\right]
$$

The ELM is a linear state-space model with known input $\{w(k)\}$ and output $\{y(k)\}$ history. Thus it can be identified by any linear identification method and then used to recover the original bilinear system matrices (Ref. [8]). The ELM approach was first presented in Refs. [8,9]. The latter relied on sinusoidal basis functions to obtain an approximate IOSR. The former introduced the concept of interaction matrices to derive a specific IOSR. In this paper this IOSR is referred to as causal IOSR because the state at the current time step is related to the current superstate which is defined in terms of past input and past output data only.

\section{Intersection Subspace (IS) Method}

If one was able to find two independent IOSRs of the form of Eq. (3), then another approach, based on the direct reconstruction of the bilinear state history, would be possible. Subspace methods have been very successful in linear system identification (see Ref. [14] for a detailed presentation) but are very general. In particular, their versions based on the intersection of two vector spaces (Ref. [15]) can be applied to reconstruct the state of any kind of system for which two relations like Eq. (3) can be obtained.

\section{Superspaces}

Assume the following IOSRs are available for the bilinear system of Eq. (1)

$$
x(k)=T_{a} z_{a}(k) \quad x(k)=T_{b} z_{b}(k)
$$

and define the following matrices

$$
\begin{aligned}
X & =\left[\begin{array}{lllll}
x\left(k_{i}\right) & x\left(k_{i}+1\right) & x\left(k_{i}+2\right) & \ldots & x\left(k_{f}\right)
\end{array}\right] \\
Z_{a} & =\left[\begin{array}{lllll}
z_{a}\left(k_{i}\right) & z_{a}\left(k_{i}+1\right) & z_{a}\left(k_{i}+2\right) & \ldots & z_{a}\left(k_{f}\right)
\end{array}\right] \\
Z_{b} & =\left[\begin{array}{lllll}
z_{b}\left(k_{i}\right) & z_{b}\left(k_{i}+1\right) & z_{b}\left(k_{i}+2\right) & \ldots & z_{b}\left(k_{f}\right)
\end{array}\right]
\end{aligned}
$$

where $k_{i}$ and $k_{f}$ are the initial and final time steps for which Eq. (7) holds. Then we can write

$$
X=T_{a} Z_{a} \quad X=T_{b} Z_{b}
$$


The row space of the (minimum-dimension) state $X$ is a subspace of the row space of $Z_{a}$ and also a subspace of the row space of $Z_{b}$. The row space of $X$ must then lie in the intersection between the row spaces of the two superspaces $Z_{a}$ and $Z_{b}$. The problem of reconstructing the state history $\{x(k)\}$ is therefore reduced to finding the intersection of two vector spaces.

\section{Intersection}

The intersection of the two vector spaces given by the row spaces of $Z_{a}$ and $Z_{b}$ is spanned by common row vectors $h_{i}$, which can be expressed as linear combinations of the rows of $Z_{a}$ or of the rows of $Z_{b}$

$$
h_{i}=a_{i}^{T} Z_{a}=b_{i}^{T} Z_{b}
$$

where $a_{i}$ and $b_{i}$ are column vectors with the corresponding linear combination coefficients. Defining

$$
R=\left[\begin{array}{ll}
Z_{a}^{T} & Z_{b}^{T}
\end{array}\right] \quad c_{i}=\left[\begin{array}{c}
a_{i} \\
-b_{i}
\end{array}\right]
$$

we can rewrite Eq. (12) as

$$
R c_{i}=0
$$

which shows that the column vectors $c_{i}$ lie in the null space of $R$ and therefore can be conveniently found by Singular Value Decomposition (SVD) as the right singular vectors associated with the zero singular values of $R$. Note that all possible pair combinations of basis vectors of the null space of $Z_{a}^{T}$ and of the null space of $Z_{b}^{T}$ satisfy Eq. (14). In general, the null space of $R$ then has dimension $m \geq n$ and another step is necessary to get a basis for the intersection subspace (i.e. $n$ linearly independent $h_{i}$ vectors). This can be easily achieved by another SVD as follows. Knowledge of $c_{i}$ 's allows one to compute the corresponding $h_{i}$ 's through Eq. (13) and either of equalities in Eq. (12). Stacking the $m$ common row vectors $h_{i}$ 's in a matrix $H$, the $n$ basis vectors $X_{i}$ 's can be found as the right singular vectors associated with the non-zero singular values of $H$. A basis for the actual bilinear state space is obtained, which means the state history of the bilinear system is now known.

\section{Direct Identification}

Once the state history is reconstructed, the identification problem is dramatically simplified and can be solved by the classic least-squares method. From Eq. (1) we can write

$$
\begin{aligned}
{\left[\begin{array}{lll}
x\left(k_{i}+1\right) & \ldots & x\left(k_{f}\right)
\end{array}\right] } & =\left[\begin{array}{lll}
A & B & N
\end{array}\right]\left[\begin{array}{ccc}
x\left(k_{i}\right) & \ldots & x\left(k_{f}-1\right) \\
u\left(k_{i}\right) & \ldots & u\left(k_{f}-1\right) \\
x\left(k_{i}\right) u\left(k_{i}\right) & \ldots & x\left(k_{f}-1\right) u\left(k_{f}-1\right)
\end{array}\right] \\
{\left[\begin{array}{lll}
y\left(k_{i}\right) & \ldots & y\left(k_{f}\right)
\end{array}\right] } & =\left[\begin{array}{ll}
C & D
\end{array}\right]\left[\begin{array}{lll}
x\left(k_{i}\right) & \ldots & x\left(k_{f}\right) \\
u\left(k_{i}\right) & \ldots & u\left(k_{f}\right)
\end{array}\right]
\end{aligned}
$$

and recover $A, B, N$ and $C$ via pseudo-inversion (Moore-Penrose)

$$
\begin{gathered}
{\left[\begin{array}{lll}
A & B & N
\end{array}\right]=\left[\begin{array}{lll}
x\left(k_{i}+1\right) & \ldots & x\left(k_{f}\right)
\end{array}\right]\left[\begin{array}{ccc}
x\left(k_{i}\right) & \ldots & x\left(k_{f}-1\right) \\
u\left(k_{i}\right) & \ldots & u\left(k_{f}-1\right) \\
x\left(k_{i}\right) u\left(k_{i}\right) & \ldots & x\left(k_{f}-1\right) u\left(k_{f}-1\right)
\end{array}\right]^{\dagger}} \\
{\left[\begin{array}{ll}
C & D
\end{array}\right]}
\end{gathered}
$$

Note that the state reconstructed by intersection is not necessarily in the original coordinate system, and so will be the identified bilinear model matrices $A, B, N, C, D$. As usual with state-space formulation, the change in coordinate system does not affect the identified model validity. Also, a sufficient richness condition for the input excitation is that the rank of the state-input matrix to be inverted in Eq. (17) is equal to $2 n+1$. 


\section{Comparison}

The comparison between the ELM and IS methods is more subtle than it appears at a first glance. In this paper, we will limit ourselves to the following considerations. The core of the IS method is state reconstruction, which then makes it trivial to estimate the dynamic system matrices. The IS method can be applied for state reconstruction directly on the bilinear model of Eq. (1) or on the ELM of Eq. (4); in the latter case, the IS method is used as a purely linear identification algorithm. The application of the IS method to the bilinear model of Eq. (1) is more direct but it requires the existence of at least two IOSRs for the bilinear model; in this paper, several IOSRs will be derived to make it possible to apply the IS method to bilinear system identification.

\section{USEFUL CONCEPTS}

Before plunging into the algebraic derivation of IOSRs, a few concepts useful throughout the rest of the paper are presented here below.

\section{Interaction Matrices}

The concept of interaction matrices is at the core of this work (Ref. [16]). The interaction matrices were originally formulated by Minh Q. Phan in the context of linear system identification of lightly-damped large flexible space structures. The dynamics of such structures can be described by the system Markov parameters. The identification of these Markov parameters can become difficult because of the very large number of system Markov parameters that must be solved for. The interaction matrix provides a mechanism to compress an infinite sequence of system Markov parameters into a finite sequence that can be easily identified from input-output measurements (Refs. [17, 18]). The compression can be exact and extremely efficient. The system Markov parameters are then recovered from the compressed Markov parameters, and remarkably, the recovery can be achieved without having to know the interaction matrix required for the compression in the first place. Later development revealed that the interaction matrix in the state-space system identification problem could be interpreted as a Kalman filter gain that is optimal with respect to the system and the (unknown) process and noise statistics embedded in the input-output data. This development led to the Observer/Kalman filter Identification (OKID) algorithm (Ref. [19]).

The interaction matrices were recently proposed for bilinear system identification in Ref. [8]. For the sake of clarity, the key steps to derive the IOSR are reported here below (for more details about the derivation, see Ref. [8]). Consider the bilinear system of Eq. (1) and add and subtract the terms $M^{\prime} y(k)$ and $M^{\prime \prime} y(k) u(k)$ where $M^{\prime}$ and $M^{\prime \prime}$ are interaction matrices, getting

$$
\begin{aligned}
x(k+1)= & A x(k)+N x(k) u(k)+B u(k)+M^{\prime} y(k)-M^{\prime} y(k)+M^{\prime \prime} y(k) u(k)-M^{\prime \prime} y(k) u(k) \\
= & A x(k)+N x(k) u(k)+B u(k)+M^{\prime} C x(k)+M^{\prime} D u(k)-M^{\prime} y(k) \\
& +M^{\prime \prime} C x(k) u(k)+M^{\prime \prime} D u^{2}(k)-M^{\prime \prime} y(k) u(k) \\
= & \left(A+M^{\prime} C\right) x(k)+\left(N+M^{\prime \prime} C\right) x(k) u(k) \\
& +\left(B+M^{\prime} D\right) u(k)-M^{\prime} y(k)+M^{\prime \prime} D u^{2}(k)-M^{\prime \prime} y(k) u(k)
\end{aligned}
$$

which can be rewritten as

$$
x(k+1)=\bar{A} x(k)+\bar{N} x(k) u(k)+\bar{B} v(k)
$$

where

$$
\begin{gathered}
\bar{A}=A+M^{\prime} C \quad \bar{N}=N+M^{\prime \prime} C \\
\bar{B}=\left[\begin{array}{lll}
B+M^{\prime} D & -M^{\prime} & \left.M^{\prime \prime} D-M^{\prime \prime}\right] \quad v(k)= \\
\\
u^{2}(k) \\
y(k) u(k)
\end{array}\right]
\end{gathered}
$$

The interaction matrices convert the bilinear model $A, B, N$ of Eq. (1a) into an equivalent bilinear model $\bar{A}, \bar{N}, \bar{B}$, Eq. (19). The observation equation, Eq. (1b), does not change. The freedom introduced by $M^{\prime}$ 
and $M^{\prime \prime}$ will be used to impose conditions to express the state at the current time step $k$ solely in terms of past input and output data. In the following, for brevity of presentation, we will terminate the propagation of Eq. (19) at time step $k+2$, but it is possible to generalize the termination at any time step $k+p$. Propagating Eq. (19) one step forward, we get

$$
\begin{aligned}
x(k+2)= & \bar{A} x(k+1)+\bar{N} x(k+1) u(k+1)+\bar{B} v(k+1) \\
= & \bar{A}^{2} x(k)+\bar{A} \bar{N} x(k) u(k)+\bar{A} \bar{B} v(k)+\bar{N} \bar{A} x(k) u(k+1) \\
& +\bar{N}^{2} x(k) u(k) u(k+1)+\bar{N} \bar{B} v(k) u(k+1)+\bar{B} v(k+1)
\end{aligned}
$$

Assuming the sum of all the terms in Eq. (22) depending on the state $x(k)$ vanish, i.e.

$$
\bar{A}^{2}+\bar{A} \bar{N} u(k)+\bar{N} \bar{A} u(k+1)+\bar{N}^{2} u(k) u(k+1)=0
$$

then Eq. (22) becomes

$$
x(k+2)=\bar{A} \bar{B} v(k)+\bar{N} \bar{B} v(k) u(k+1)+\bar{B} v(k+1)
$$

and can be rewritten in the form of an IOSR as

$$
x(k+2)=T_{2} z_{2}(k+2)
$$

where

$$
T_{2}=\left[\begin{array}{lll}
\bar{A} \bar{B} & \bar{N} \bar{B} & \bar{B}
\end{array}\right] \quad z_{2}(k+2)=\left[\begin{array}{c}
v(k) \\
v(k) u(k+1) \\
v(k+1)
\end{array}\right]
$$

and the subscript 2 remarks that the state depends on input-output data two steps back in time. Equation (25), or equivalently Eq. (24), expresses the state of the the bilinear model of Eq. (1) in terms of previous input-output data only. Shifting the time index backward by 2, Eq. (25) takes the same form as Eq. (3).

As mentioned, Eq. (22) can be propagated further in time, leading to a higher-order $(p>2)$ IOSR of the form

$$
x(k)=T_{p} z_{p}(k)
$$

In Ref. [8], the general pattern to construct the superstate $z_{p}(k)$ for the above IOSR from input-output data is derived. Generalizing Eq. (23), the condition to be satisfied to get to Eq. (27) is that the sum of all the terms depending on $x(k)$ in the propagated equation vanish. This condition will be symbolically indicated as

$$
\mathcal{S}_{p}(\bar{A}, \bar{N},\{u(k) \ldots u(k+p-1)\})=0
$$

and for $p=2$ it takes the explicit form of Eq. (23).

\section{Exact Input-Output-to-State Representations and Ideal Bilinear Systems}

The condition for Eq. (23) to be identically satisfied for any input history $\{u(k)\}$ is

$$
\bar{A}^{2}=\bar{A} \bar{N}=\bar{N} \bar{A}=\bar{N}^{2}=0
$$

To identically satisfy the generalized condition of Eq. (28), it is necessary that all the possible products of order $p$ of matrices $\bar{A}$ and $\bar{N}$ are zero, which in this paper is more compactly indicated as

$$
\mathcal{C}_{p}(\bar{A}, \bar{N})=0
$$

In Refs. [8,13] the existence of interaction matrices $M^{\prime}$ and $M^{\prime \prime}$ such that Eq. (30) is satisfied was only postulated. It is indeed a matter of fact that there exist bilinear systems for which Eq. (30) holds for some $p$. In this paper, they are referred to as ideal bilinear systems and a couple of examples are given in the Examples section. For an ideal bilinear system it is thus possible to get an exact IOSR and achieve (apart from noise) exact identification via the ELM or the IS methods. It is worth remarking that the concept of ideal bilinear system is used only for the purpose of theoretical considerations, and the techniques developed in this paper apply to arbitrary bilinear systems of the form of Eq. (1), as proven by the next theorem and shown in the final examples. 


\section{Convergence of Non-Ideal Bilinear Systems}

The following theorem provides rigorous justification for the application to any bilinear system of ELM and IS identification methods based on IOSRs derived via interaction matrices.

Theorem. Given any bilinear system of the form of Eq. (1), if $(A, C)$ is a detectable pair then there exist interaction matrices $M^{\prime}$ and $M^{\prime \prime}$ and a value $\gamma$ such that, for $|u(k)|<\gamma$ for all $k$, Eq. (28) is asymptotically satisfied as $p$ increases.

In particular, the theorem ensures that, even though Eq. (30) is not identically satisfied, the resulting approximation error in the IOSR converges to zero as the order $p$ is increased, provided the input is kept within a certain threshold. The proof has its roots in the similarity between the present problem and the work of Bouazza et al. (Ref. [20]), who demonstrated the convergence of a Luenberger-like observer for a class of discrete-time nonlinear systems which can be shown to include bilinear systems of the form of Eq. (1).

Proof. Consider Eq. (1a). Introducing interaction matrices $M^{\prime}$ and $M^{\prime \prime}$, we were able to write Eq. (19), which was then propagated forward by one time step to obtain $x(k+2)$ in Eq. (22). Note that the terms containing the state $x(k)$ in Eq. (22), which were eliminated via the condition of Eq. (23), do not involve the input $v(k)$. Also, note that the terms depending on $v(k)$ in Eq. (22) do not contain the state $x(k)$. These observations can be generalized to propagation in time up to any step $k+p$. Since the objective is to prove convergence to zero of the sum of the terms in Eq. (23) and in the more general Eq. (28), it is sufficient to study the convergence to the origin of the following dynamic system

$$
\hat{x}(k+1)=\bar{A} \hat{x}(k)+\bar{N} \hat{x}(k) u(k)
$$

as explained in what follows. Propagating Eq. (31) forward, we obtain

$$
\hat{x}(k+p)=\mathcal{S}_{p}(\bar{A}, \bar{N},\{u(k) \ldots u(k+p-1)\}) \hat{x}(k)
$$

If we prove that, for any arbitrary $\hat{x}(k), \hat{x}(k+p)$ converges to zero as $p$ increases, then convergence of $\mathcal{S}_{p}(\bar{A}, \bar{N},\{u(k) \ldots u(k+p-1)\})$ to zero will follow.

Recall the definition of $\bar{A}$, Eq. (20) and assume $A$ and $C$ form a detectable pair. Then there exists a positive definite matrix $S$ such that

$$
\bar{A}^{T} S \bar{A}+I=S
$$

Define the weighted norm

$$
X(k)=\hat{x}^{T}(k) S \hat{x}(k)
$$

which will be the measure of convergence to zero of $\hat{x}(k)$. Even more conveniently, define the increment of the above weighted norm

$$
\Delta X(k)=X(k+1)-X(k)
$$

Plugging Eq. (31) into the first term of Eq. (35) and Eq. (33) into the second term we obtain

$$
\begin{aligned}
\Delta X(k) & =\hat{x}^{T}(k+1) S \hat{x}(k+1)-\hat{x}^{T}(k) S \hat{x}(k) \\
& =(\bar{A} \hat{x}(k)+\bar{N} \hat{x}(k) u(k))^{T} S(\bar{A} \hat{x}(k)+\bar{N} \hat{x}(k) u(k))-\hat{x}^{T}(k)\left(\bar{A}^{T} S \bar{A}+I\right) \hat{x}(k) \\
& =u^{2}(k) \hat{x}^{T}(k) \bar{N}^{T} S \bar{N} \hat{x}(k)+2 u(k) \hat{x}^{T}(k) \bar{A}^{T} S \bar{N} \hat{x}(k)-\hat{x}^{T}(k) \hat{x}(k) \\
& =-\|\hat{x}(k)\|^{2}\left(-\frac{1}{\|\hat{x}(k)\|^{2}} u^{2}(k) \hat{x}^{T}(k) \bar{N}^{T} S \bar{N} \hat{x}(k)-\frac{1}{\|\hat{x}(k)\|^{2}} 2 u(k) \hat{x}^{T}(k) \bar{A}^{T} S \bar{N} \hat{x}(k)+1\right)
\end{aligned}
$$

from which it is clear that $X(k)$ will converge to zero if the term in parentheses in Eq. (36) is positive, i.e. if

$$
\frac{1}{\|\hat{x}(k)\|^{2}} u^{2}(k) \hat{x}(k)^{T} \bar{N}^{T} S \bar{N} \hat{x}(k)+\frac{1}{\|\hat{x}(k)\|^{2}} 2 u(k) \hat{x}(k)^{T} \bar{A}^{T} S \bar{N} \hat{x}(k)<1
$$


By basic matrix norm properties, the following inequalities follow

$$
\begin{gathered}
u^{2}(k) \hat{x}(k)^{T} \bar{N}^{T} S \bar{N} \hat{x}(k) \leq\left\|u^{2}(k) \hat{x}(k)^{T} \bar{N}^{T} S \bar{N} \hat{x}(k)\right\| \leq u^{2}(k)\|\hat{x}(k)\|^{2}\|\bar{N}\|^{2}\|S\| \\
2 u(k) \hat{x}(k)^{T} \bar{A}^{T} S \bar{N} \hat{x}(k) \leq\left\|2 u(k) \hat{x}(k)^{T} \bar{A}^{T} S \bar{N} \hat{x}(k)\right\| \leq 2|u(k)|\|\hat{x}(k)\|^{2}\|\bar{A}\|\|\bar{N}\|\|S\|
\end{gathered}
$$

and allow us to rewrite the convergence condition, Eq. (37), as

$$
u^{2}(k)\|\bar{N}\|^{2}|| S||+2|u(k)|\|\bar{A}\|\|\bar{N}\|\|S\|<1
$$

which is always possible to satisfy by choosing $|u(k)|<\gamma$ for all $k$, with sufficiently small $\gamma$. By Eq. (34), convergence of $X(k)$ implies convergence of $\hat{x}(k)$, as desired.

The above theorem is a fundamental finding that provides theoretical justification of the overall approach to bilinear system identification proposed here, and in particular of the application of the concept of interaction matrices to bilinear systems. In the rest of the paper, we will sometimes refer to exact IOSRs and approximate IOSRs to remark the fact that not all bilinear systems satisfy Eq. (28) identically, but it must be kept in mind that increasing the IOSR order $p$ will make the approximation error converge to zero. It is worth remarking that the requirement of bounded input concerns the excitation to get input-output data for identification. Once the bilinear model is identified, it can be used to predict the output of the real bilinear system for inputs higher than the above mentioned bound.

\section{INPUT-OUTPUT-TO-STATE REPRESENTATIONS}

To implement the IS identification method it is necessary to have two independent Input-Output-to-State Representations (IOSRs). Another IOSRs is necessary, other than the one derived above, Eq. (27). Additionally, even when applying the ELM method, some IOSRs can be more favorable than others for certain bilinear systems. Thus it is of interest to find other IOSRs like Eq. (27), i.e. other linear relations between the bilinear system state and a superstate made of input-output data only.

\section{Causal Representation}

A causal IOSR is here defined as a representation of the form of Eq. (3) where the state depends on past (and current, at most) input-output data only, i.e. $x(k)$ depends on $u(i)$ and $y(i)$ with $i \leq k$. The example of IOSR derived above is causal. Its key equations are rewritten as follows introducing the subscript $c$ where needed to remark the causality of the representation. Equation (19) becomes

$$
x(k+1)=\bar{A}_{c} x(k)+\bar{N}_{c} x(k) u(k)+\bar{B}_{c} v(k)
$$

where

$$
\begin{gathered}
\bar{A}_{c}=A+M_{c}^{\prime} C \quad \bar{N}_{c}=N+M_{c}^{\prime \prime} C \\
\bar{B}_{c}=\left[B+M_{c}^{\prime} D-M_{c}^{\prime} M_{c}^{\prime \prime} D-M_{c}^{\prime \prime}\right] \quad v_{c}(k)=\left[\begin{array}{c}
u(k) \\
y(k) \\
u^{2}(k) \\
y(k) u(k)
\end{array}\right]
\end{gathered}
$$

and the causal IOSR of Eq. (27) is denoted by

$$
x(k)=T_{p, c} z_{p, c}(k)
$$

where the superstate $z_{p, c}(k)$ depends on input-output data at steps $k-1, k-2, \ldots, k-p$ only. Letting $n C k=\left(\begin{array}{l}n \\ k\end{array}\right)$ denote the combinations of $k$ out of $n$ terms, commonly referred to as $n$-choose- $k$, the general pattern for the entries of the column vector $z_{p, c}(k+p)$ is (Ref. [8]):

$$
-v_{c}(k), v_{c}(k+1), \ldots \text { to } v_{c}(k+p-1)
$$


- $v_{c}(k)$ multiplied with products of $u(k+1)$ to $u(k+p-1)$ in all possible combinations $(p-1) C 1$, $(p-1) C 2, \ldots,(p-1) C(p-1)$ of $\{u(k+1), u(k+2), \ldots, u(k+p-1)\}$

- $v_{c}(k+1)$ multiplied with products of $u(k+2)$ to $u(k+p-1)$ in all possible combinations $(p-2) C 1$, $(p-2) C 2, \ldots,(p-2) C(p-2)$ of $\{u(k+2), u(k+3), \ldots, u(k+p-1)\}$

- $v_{c}(k+p-3)$ multiplied with products of $u(k+p-2)$ to $u(k+p-1)$ in all possible combinations $2 C 1,2 C 2$ of $\{u(k+3), u(k+4), \ldots, u(k+p-1)\}$

- $v_{c}(k+p-2)$ multiplied with $1 C 1$ of $u(k+p-1)$, which of course is $u(k+p-1)$

To obtain an expression for $z_{p, c}(k)$, we simply shift the time indices of $z_{p, c}(k+p)$ backwards by $p$ time steps.

\section{Anticausal Representation}

An anticausal IOSR is here defined as a representation of the form of Eq. (3) where the state depends on future (and current, at most) input-output data only, i.e. $x(k)$ depends on $u(i)$ and $y(i)$ with $i \geq k$.

Rewrite Eq. (1) as

$$
\begin{aligned}
& x(k)=A^{-1} x(k+1)-A^{-1} N x(k) u(k)-A^{-1} B u(k) \\
& y(k)=C x(k)+D u(k)
\end{aligned}
$$

Let $M_{a}^{\prime}$ and $M_{a}^{\prime \prime}$ be another pair of interaction matrices, where the subscript $a$ stands for anticausal, and add and subtract the terms $M_{a}^{\prime} y(k+1)$ and $M_{a}^{\prime \prime} y(k) u(k)$ in (45a), getting

$$
\begin{aligned}
x(k)= & A^{-1} x(k+1)-A^{-1} N x(k) u(k)-A^{-1} B u(k) \\
& +M_{a}^{\prime} y(k+1)-M_{a}^{\prime} y(k+1)+M_{a}^{\prime \prime} y(k) u(k)-M_{a}^{\prime \prime} y(k) u(k) \\
= & A^{-1} x(k+1)-A^{-1} N x(k) u(k)-A^{-1} B u(k)+M_{a}^{\prime} C x(k+1)+M_{a}^{\prime} D u(k+1) \\
& -M_{a}^{\prime} y(k+1)+M_{a}^{\prime \prime} C x(k) u(k)+M_{a}^{\prime \prime} D u^{2}(k)-M_{a}^{\prime \prime} y(k) u(k) \\
= & \left(A^{-1}+M_{a}^{\prime} C\right) x(k+1)+\left(-A^{-1} N+M_{a}^{\prime \prime} C\right) x(k) u(k) \\
& -A^{-1} B u(k)+M_{a}^{\prime} D u(k+1)-M_{a}^{\prime} y(k+1)+M_{a}^{\prime \prime} D u^{2}(k)-M_{a}^{\prime \prime} y(k) u(k)
\end{aligned}
$$

which can be rewritten as

$$
x(k)=\bar{A}_{a} x(k+1)+\bar{N}_{a} x(k) u(k)+\bar{B}_{a} v_{a}(k+1)
$$

where

$$
\begin{aligned}
& \bar{A}_{a}=A^{-1}+M_{a}^{\prime} C \quad \bar{N}_{a}=-A^{-1} N+M_{a}^{\prime \prime} C \\
& \bar{B}_{a}=-\left[\begin{array}{lllll}
A^{-1} B & -M_{a}^{\prime} D & M_{a}^{\prime} & -M_{a}^{\prime \prime} D & M_{a}^{\prime \prime}
\end{array}\right] \quad v_{a}(k+1)=\left[\begin{array}{c}
u(k) \\
u(k+1) \\
y(k+1) \\
u^{2}(k) \\
y(k) u(k)
\end{array}\right]
\end{aligned}
$$

Rewriting Eq. (47) for the next time step

$$
x(k+1)=\bar{A}_{a} x(k+2)+\bar{N}_{a} x(k+1) u(k+1)+\bar{B}_{a} v_{a}(k+2)
$$

and plugging Eq. (47) and Eq. (50) into Eq. (47) itself, we get

$$
\begin{aligned}
x(k)= & \bar{A}_{a}\left(\bar{A}_{a} x(k+2)+\bar{N}_{a} x(k+1) u(k+1)+\bar{B}_{a} v_{a}(k+2)\right) \\
& +\bar{N}_{a}\left(\bar{A}_{a} x(k+1)+\bar{N}_{a} x(k) u(k)+\bar{B}_{a} v_{a}(k+1)\right) u(k)+\bar{B}_{a} v_{a}(k+1) \\
= & \bar{A}_{a}^{2} x(k+2)+\bar{A}_{a} \bar{N}_{a} x(k+1) u(k+1)+\bar{A}_{a} \bar{B}_{a} v_{a}(k+2)+\bar{N}_{a} \bar{A}_{a} x(k+1) u(k) \\
& +\bar{N}_{a}^{2} x(k) u(k)^{2}+\bar{N}_{a} \bar{B}_{a} v_{a}(k+1) u(k)+\bar{B}_{a} v_{a}(k+1)
\end{aligned}
$$


Assuming, similarly to Eq. (29),

$$
\bar{A}_{a}^{2}=\bar{A}_{a} \bar{N}_{a}=\bar{N}_{a} \bar{A}_{a}=\bar{N}_{a}^{2}=0
$$

we get from Eq. (51)

$$
x(k)=\bar{A}_{a} \bar{B}_{a} v_{a}(k+2)+\bar{N}_{a} \bar{B}_{a} v_{a}(k+1) u(k)+\bar{B}_{a} v_{a}(k+1)
$$

which we can rewrite as

$$
x(k)=T_{2, a} z_{2, a}(k)
$$

where

$$
T_{2, a}=\left[\begin{array}{lll}
\bar{A}_{a} \bar{B}_{a} & \bar{N}_{a} \bar{B}_{a} & \bar{B}_{a}
\end{array}\right] \quad z_{2, a}(k)=\left[\begin{array}{c}
v_{a}(k+2) \\
v_{a}(k+1) u(k) \\
v_{a}(k+1)
\end{array}\right]
$$

Equation (54), or equivalently Eq. (53), expresses the state of the bilinear model in terms of current and future input-output data only, and is an anticausal IOSR specialized for $p=2$.

The rationale in the above derivation is to find an expression for the state written in terms of input-output data only, via an assumption similar to the one made for the causal representation, i.e. eliminating the dependance of $x(k)$ on the states at any other time steps. The aim is the same as in the causal representation, however the derivation is less straightforward. To better show the approach to find higher-order anticausal IOSRs, the derivation for $p=3$ is presented as well. After rewriting Eq. (50) for the next time step

$$
x(k+2)=\bar{A}_{a} x(k+3)+\bar{N}_{a} x(k+2) u(k+2)+\bar{B}_{a} v_{a}(k+3)
$$

plug Eqs. $(47,50,56)$ into Eq. (51) to get an expression for $x(k)$ whose (unknown) state-dependent terms all contain $3^{\text {rd }}$-order products of matrices $\bar{A}_{a}$ and $\bar{N}_{a}$

$$
\begin{aligned}
x(k)= & \bar{A}_{a}^{3} x(k+3)+\bar{A}_{a}^{2} \bar{N}_{a} x(k+2) u(k+2)+\bar{A}_{a} \bar{N}_{a} \bar{A}_{a} x(k+2) u(k+1) \\
& +\bar{N}_{a} \bar{A}_{a}^{2} x(k+2) u(k)+\bar{A}_{a} \bar{N}_{a}^{2} x(k+1) u^{2}(k+1)+\bar{N}_{a} \bar{A}_{a} \bar{N}_{a} x(k+1) u(k+1) u(k) \\
& +\bar{N}_{a}^{2} \bar{A}_{a} x(k+1) u^{2}(k)+\bar{N}_{a}^{3} x(k) u^{3}(k)+\bar{A}_{a}^{2} \bar{B}_{a} v_{a}(k+3) \\
& +\bar{A}_{a} \bar{N}_{a} \bar{B}_{a} v_{a}(k+2) u(k+1)+\bar{N}_{a} \bar{A}_{a} \bar{B}_{a} v_{a}(k+2) u(k)+\bar{A}_{a} \bar{B}_{a} v_{a}(k+2) \\
& +\bar{N}_{a}^{2} \bar{B}_{a} v_{a}(k+1) u^{2}(k)+\bar{N}_{a} \bar{B}_{a} v_{a}(k+1) u(k)+\bar{B}_{a} v_{a}(k+1)
\end{aligned}
$$

Assuming that the terms with matrix products given by all the possible combinations of $\bar{A}$ and $\bar{N}$ of $3^{\text {rd }}$ order vanish, we get

$$
\begin{aligned}
x(k)= & \bar{A}_{a}^{2} \bar{B}_{a} v_{a}(k+3)+\bar{A}_{a} \bar{N}_{a} \bar{B}_{a} v_{a}(k+2) u(k+1)+\bar{N}_{a} \bar{A}_{a} \bar{B}_{a} v_{a}(k+2) u(k) \\
& +\bar{A}_{a} \bar{B}_{a} v_{a}(k+2)+\bar{N}_{a}^{2} \bar{B}_{a} v_{a}(k+1) u^{2}(k)+\bar{N}_{a} \bar{B}_{a} v_{a}(k+1) u(k)+\bar{B}_{a} v_{a}(k+1)
\end{aligned}
$$

which can be rewritten as

$$
x(k)=T_{3, a} z_{3, a}(k)
$$

where

$$
\begin{aligned}
& T_{3, a}=\left[\begin{array}{lllllllll}
\bar{A}_{a}^{2} \bar{B}_{a} & \bar{A}_{a} \bar{N}_{a} \bar{B}_{a} & \bar{N}_{a} \bar{A}_{a} \bar{B}_{a} & \bar{N}_{a}^{2} \bar{B}_{a} & \bar{A}_{a} \bar{B}_{a} & \bar{N}_{a} \bar{B}_{a} & \bar{B}_{a}
\end{array}\right] \\
& z_{3, a}(k)=\left[\begin{array}{c}
v_{a}(k+3) \\
v_{a}(k+2) u(k+1) \\
v_{a}(k+2) u(k) \\
v_{a}(k+1) u^{2}(k) \\
v_{a}(k+2) \\
v_{a}(k+1) u(k) \\
v_{a}(k+1)
\end{array}\right]
\end{aligned}
$$

Following the same approach, anticausal IOSRs can be obtained for any arbitrary value of $p$ and are denoted by

$$
x(k)=T_{p, a} z_{p, a}(k)
$$

where the superstate $z_{p, a}(k)$ is defined as the column vector with the following entries 


$$
\begin{aligned}
& -v_{a}(k+1) u^{i}(k) \text { for all } i=0,1, \ldots, p-1 \\
& -v_{a}(k+2) u^{i_{1}}(k) u^{i_{2}}(k+1) \text { for all } i_{1}, i_{2} \geq 0 \text { and } i_{1}+i_{2} \leq p-2 \\
& \quad \cdot \\
& \quad \cdot \\
& -v_{a}(k+r) u^{i_{1}}(k) u^{i_{2}}(k+1) \ldots u^{i_{p}}(k+p-r) \text { for all } i_{j} \geq 0 \text { and } \sum_{j=1}^{r} i_{j} \leq p-r \\
& \quad \cdot \\
& \quad \cdot \\
& -v_{a}(k+p)
\end{aligned}
$$

and depends on input-output data at steps $k, k+1, \ldots, k+p$ only. Note that the above definition of superstate $z_{p, a}(k)$ with $v_{a}(k)$ given by Eq. (49) leads to some redundancy in the entries of $z_{p, a}(k)$, which is suggested to be eliminated when implementing the desired identification algorithm.

It is worth remarking that the condition for a bilinear system to have an exact anticausal IOSR is formally the same as for the causal IOSR, but it involves $\bar{A}_{a}$ and $\bar{N}_{a}$ instead of $\bar{A}_{c}$ and $\bar{N}_{c}$

$$
\mathcal{C}_{p}\left(\bar{A}_{a}, \bar{N}_{a}\right)=0
$$

A bilinear system ideal in backward sense is defined as a system satisfying Eq. (62) for some $p$.

To be rigorous, the previous theorem about convergence for increasing $p$ refers to the causal IOSR and its extension the anticausal IOSR would need modifying the proof. Numerical evidence indeed shows convergence of the anticausal IOSR; however, the extension of the theorem is unnecessary thanks to the mixedanticausal IOSR that will be presented next.

\section{Considerations on Causal and Anticausal Representations}

Having so far developed two IOSRs only, no choice is possible when using the IS method. In contrast, the ELM method requires one IOSR only, therefore asking which IOSR is preferred is of interest.

Consider two extreme examples. If the system to be identified was an ideal bilinear model (in forward sense), then the causal IOSR would be the preferred choice since, being exact, it would in turn give an exact ELM and exact identification (apart from noise). Similarly, if the system in question was an ideal bilinear model in backward sense, then the anticausal IOSR would have to be chosen. A more realistic example concerns a non-ideal bilinear system, whose causal (anticausal) IOSR converges to the actual dynamics faster than its anticausal (causal) IOSR as $p$ increases. A preferred choice would still exist and be the causal (anticausal) IOSR. An a priori optimal choice is not possible. Obviously, it is very hard to know how close a system is to being ideal before its identification. Still, having different IOSRs at our disposal is helpful, allowing us to try different ELMs and then choose the one converging faster, with apparent computational advantages.

It is worth remarking that when the IS identification method is used, the order $p$ of the causal and anticausal representation can be different $\left(p_{c} \neq p_{a}\right)$. The ideal situation would be to have an ideal bilinear system in both forward and backward sense, which is a rare (if at all possible) occurrence. Four interaction matrices $\left(M_{c}^{\prime}\right.$, $M_{c}^{\prime \prime}, M_{a}^{\prime}, M_{a}^{\prime \prime}$ ) are required. One non-exact IOSR is sufficient to make the IS method approximate (obviously, the larger $p$, the more accurate the identification, thanks to the above theorem about IOSR convergence).

Based on these considerations, it makes sense to ask whether other, more favorable, IOSRs can be found. Here two more examples are provided and the resulting representations are referred to as mixed since they relate the current state to both past and future input-output data. The remarkable feature of these mixed representations is that each of them needs three interaction matrices, and when used together with a causal or anticausal representation the overall requirement for the IS method to be exact is relaxed to the existence of three interaction matrices only. 


\section{Mixed-Anticausal Representations}

Start from Eq. (47) and work on its right-hand side replacing $x(k+1)$ with Eq. (50) and $x(k)$ with Eq. (44) to get

$$
\begin{aligned}
x(k)= & \bar{A}_{a}^{2} x(k+2)+\bar{A}_{a} \bar{N}_{a} x(k+1) u(k+1) \\
& +\bar{A}_{a} \bar{B}_{a} v_{a}(k+2)+\bar{N}_{a} \bar{T}_{p_{c}, c} z_{p_{c}, c}(k) u(k)+\bar{B}_{a} v_{a}(k+1)
\end{aligned}
$$

Replace $x(k+1)$ in Eq. (63) with Eq. (44) shifted forward by one time step, getting

$$
\begin{aligned}
x(k)= & \bar{A}_{a}^{2} x(k+2)+\bar{A}_{a} \bar{N}_{a} T_{p_{c}, c} z_{p_{c}, c}(k+1) u(k+1) \\
& +\bar{A}_{a} \bar{B}_{a} v_{a}(k+2)+\bar{N}_{a} T_{p_{c}, c} z_{p_{c}, c}(k) u(k)+\bar{B}_{a} v_{a}(k+1)
\end{aligned}
$$

Observe that the only state-dependent term on the right-hand side of Eq. (64) is multiplied by $\bar{A}_{a}^{2}$. If $n=2$, it is sufficient that $A^{-1}$ and $C$ form an observable pair to guarantee the existence of an interaction matrix $M_{a}^{\prime}$ such that $\bar{A}_{a}^{2}$ vanishes. Note that interaction matrix $M_{a}^{\prime \prime}$ becomes unnecessary, therefore $\bar{N}_{a}=N_{a}$. If $n>2$, the above derivation can be continued replacing $x(k+2)$ with Eq. (56) and taking care of the resulting terms as done above, getting an equation for $x(k)$ with the only state-dependent term multiplied by $\bar{A}_{a}^{3}$, again guaranteed to be zero if $\left(A^{-1}, C\right)$ is an observable pair. The resulting IOSR is denoted as

$$
x(k)=T_{p_{c}, p_{a}, m a} z_{p_{c}, p_{a}, m a}(k)
$$

where, for the case $n=2, p_{a}$ can be 2 and $T_{p_{c}, p_{a}, m a}$ and $z_{p_{c}, p_{a}, m a}(k)$ specialize to

$$
T_{p_{c}, 2, m a}=\left[\begin{array}{llll}
\bar{A}_{a} \bar{N}_{a} T_{p_{c}, c} & \bar{N}_{a} T_{p_{c}, c} & \bar{A}_{a} \bar{B}_{a} & \bar{B}_{a}
\end{array}\right] \quad z_{p_{c}, 2, m a}(k)=\left[\begin{array}{c}
z_{p_{c}, c}(k+1) u(k+1) \\
z_{p_{c}, c}(k) u(k) \\
v_{a}(k+2) \\
v_{a}(k+1)
\end{array}\right]
$$

More in general,

$$
z_{p_{c}, p_{a}, m a}(k)=\left[\begin{array}{c}
z_{p_{c}, c}\left(k+p_{a}-1\right) u\left(k+p_{a}-1\right) \\
\ldots \\
z_{p_{c}, c}(k) u(k) \\
v_{a}\left(k+p_{a}\right) \\
\ldots \\
v_{a}(k+1)
\end{array}\right]
$$

and the subscript $m a$ stands for mixed-anticausal to distinguish this IOSR from its counterpart derived below. The IOSR above is mixed because it depends on both past and future input-output data. The definition of its superstate features a heavier contribution from future input-output data, hence the $a$ in the subscript. Also, note that the definition of $v_{a}(k)$ given in Eq. (49) leads to some redundancy in the entries of $z_{p_{c}, p_{a}, m a}(k)$ in Eq. (67), which is not difficult to eliminate when implementing the desired identification algorithm.

\section{Mixed-Causal Representation}

With a similar approach, a relation symmetric to Eq. (65) can be derived

$$
x(k)=T_{p_{c}, p_{a}, m c} z_{p_{c}, p_{a}, m c}(k)
$$

where

$$
z_{p_{c}, p_{a}, m c}(k)=\left[\begin{array}{c}
z_{p_{a}, a}\left(k-p_{c}\right) u\left(k-p_{c}\right) \\
\cdots \\
z_{p_{a}, a}(k-1) u(k-1) \\
v_{c}\left(k-p_{c}\right) \\
\cdots \\
v_{c}(k-1)
\end{array}\right]
$$

Again, note that the definition of superstate $z_{p, a}(k)$ of Eq. (61) with $v_{a}(k)$ given by Eq. (49) leads to some redundancy in the entries of $z_{p_{c}, p_{a}, m c}(k)$, which is suggested to be eliminated when implementing the desired identification algorithm. 


\section{Considerations on Mixed Representations}

The mixed-anticausal IOSR can be of great advantage when used in the IS identification method. As already highlighted, for the mixed-anticausal IOSR to be exact, it is necessary for the bilinear system to be ideal only in forward sense, despite its mixed nature. This means that an ideal bilinear system (in forward sense) can be identified exactly with a version of the IS method based on causal and mixed-anticausal IOSRs. The only condition imposed on the backward system, Eq. (47), is that $\left(A^{-1}, C\right)$ is an observable pair. $M_{a}^{\prime \prime}$ is no longer required to exist and the overall requirements on interaction matrix existence are significantly relaxed. For the sake of clarity, it is worth remarking that the matrix $M_{a}^{\prime}$ making the mixed-anticausal IOSR exact is in general different from the matrix $M_{a}^{\prime}$ necessary for the anticausal IOSR to be exact. The mixedcausal representation is complementary to the mixed-anticausal IOSR in the sense that, when used together with the anticausal IOSR in the IS identification method, it requires the bilinear system to be ideal only in backward sense for the identification to be exact. As a conclusion, the existence of $M_{c}^{\prime}, M_{a}^{\prime}$ and $M_{a}^{\prime \prime}$ only is required.

The advantage does not disappear for more general non-ideal bilinear systems. Obviously, if the system turns out to converge faster in its forward (backward) formulation, then the IS method will benefit from the choice of causal and mixed-anticausal (anticausal and mixed-causal) IOSRs. To be noted is the fact that the dimension of the superstate in the above mixed IOSRs is larger than in purely causal or anticausal IOSRs. There would be no point in using a mixed representation in the ELM identification method.

\section{ALGORITHMS}

In this section, a step-by-step description of the methods proposed above for discrete-time bilinear system identification is provided. The data necessary to run the algorithms is the time history of the measured input $u(k)$ and output $y(k), k=0,1, \ldots, l-1$.

\section{Equivalent Linear Model (ELM) Method}

1. Construction of ELM Input. Form $w(k)$ time history, Eq. (6), for $k=k_{i}, k_{i}+1, \ldots, k_{f}$ choosing for $z(k)$ an IOSR among the following

i Causal IOSR, Eq. (44)

ii Anticausal IOSR, Eq. (61)

The order $p_{c}$ or $p_{a}$ of the selected IOSR must be chosen as well, typically greater than the assumed order of the system $n$.

2. Identification of ELM. Identify the ELM of Eq. (4) by any linear system identification technique (e.g. the superspace method described in Ref. [8] or the intersection subspace method presented in this paper with modified causal and anticausal IOSRs, which are significantly simplified for a linear system). The order $n_{i d}$ of the identified ELM will also be the order of the identified bilinear model.

3. Reconstruction of State History. Simulate the identified ELM driven by the input $w(k)$ to reconstruct the state history $x(k)$ for $k=k_{i}, k_{i}+1, \ldots, k_{f}$; since the initial state is unknown, discard the reconstructed samples corresponding to the initial transient.

4. Estimation of Bilinear System Matrices. Use the reconstructed state history $x(k)$ to form the leastsquares problems of Eqs. $(15,16)$, with $k_{i}$ now equal to a time step after the initial transient is over, and use Eqs. $(17,18)$ to estimate $A, N, B, C, D$.

Note that two variations of steps 3 and 4 to complete the estimation of the bilinear model matrices are given in Ref. [8]. 


\section{Intersection Subspace (IS) Method}

1. Construction of Superspaces. Form matrices $Z_{a}$ and $Z_{b}$, Eqs. $(9,10)$, choosing for $z_{a}(k)$ and $z_{b}(k)$ two IOSRs among the following

i Causal IOSR, Eq. (44)

ii Anticausal IOSR, Eq. (61)

iii Mixed-causal IOSR, Eqs. $(65,67)$

iv Mixed-anticausal IOSR, Eqs. $(68,69)$

The order $p_{c}$ and $p_{a}$ of the selected IOSRs must be chosen as well, typically greater than the assumed $n$ (note that $p_{c}$ can be chosen to be different from $p_{a}$ ).

2. Intersection of Superspaces. Take the SVD of $R=\left[\begin{array}{ll}Z_{a}^{T} & Z_{b}^{T}\end{array}\right]$ and select the right singular vectors (call them $c_{i}, i=1, \ldots, m$ ) corresponding to the zero singular values of $R$.

3. Construction of Basis for State History Space. Extract from vectors $c_{i}$ the first entries corresponding to vectors $a_{i}$, Eq. (13) and use them to compute row vectors $h_{i}=a_{i}^{T} Z_{a}, i=1, \ldots, m$.

4. Reduction of Basis to Linear Independent Vectors. Stack vectors $h_{i}, i=1, \ldots, m$ in a matrix $H$, take the SVD of $H$ and select its right singular vectors (call them $x_{i}, i=1, \ldots, n_{i d}$ ) corresponding to the non-zero singular values, whose number $n_{i d}$ will then be the order of the identified bilinear model.

5. Estimation of Bilinear System Matrices. Transpose column vectors $x_{i}$ to get row vectors, each of them representing the time history of the $i^{\text {th }}$ state variable of the identified model. Use vectors $x_{i}$ to form the least-squares problems of Eqs. $(17,18)$ and use Eqs. $(15,16)$ to estimate $A, N, B, C, D$.

\section{EXAMPLES}

Several numerical examples are provided to show the correctness and main features of the different identification algorithms that can be devised based on the IOSRs derived in this paper. In each example, measured input-output data are simulated by generating a random input sequence $\{u(k)\}$ of 1000 samples (from a uniform distribution between -0.5 and 0.5 ) and using it to obtain the output history $\{y(k)\}$ via the state-space model equations of the system to be identified. $\{u(k)\}$ and $\{y(k)\}$ sequences are then used to execute the chosen identification algorithm. Finally, another input sequence $\{u(k)\}$ is generated (independently from the one used for identification) and used to drive both the original system and the identified model. The difference in their outputs, also known as prediction error, is analyzed as a metric to gauge how accurate the identification algorithm was.

For detailed examples and comments on the ELM identification method based on the causal IOSR, see Ref. [8]. The following bilinear systems are used in the next examples as prototypes of ideal and arbitrary bilinear systems.

System I (ideal in forward sense)

$$
A=\left[\begin{array}{cc}
0 & 0.5 \\
0.5 & -0.5
\end{array}\right] \quad B=\left[\begin{array}{l}
1 \\
2
\end{array}\right] \quad N=\left[\begin{array}{cc}
0 & 1 \\
-1 & 1
\end{array}\right] \quad C=\left[\begin{array}{ll}
0 & 1
\end{array}\right] \quad D=0
$$

It is possible to verify that the following $M_{c}^{\prime}$ and $M_{c}^{\prime \prime}$ satisfy Eq. (29). See Ref. [8] for more details.

$$
M_{c}^{\prime}=\left[\begin{array}{c}
-0.5 \\
0.5
\end{array}\right] \quad M_{c}^{\prime \prime}=\left[\begin{array}{l}
-1 \\
-1
\end{array}\right]
$$

System II (ideal in backward sense)

$$
A=\left[\begin{array}{cc}
0.5 & 0.5 \\
0.5 & 0
\end{array}\right] \quad B=\left[\begin{array}{l}
1 \\
2
\end{array}\right] \quad N=\left[\begin{array}{cc}
0.5 & -1 \\
0 & -0.5
\end{array}\right] \quad C=\left[\begin{array}{ll}
0 & 1
\end{array}\right] \quad D=0
$$


No pair $M_{c}^{\prime}, M_{c}^{\prime \prime}$ for which Eq. (30) is satisfied exists. However, the following $M_{a}^{\prime}$ and $M_{a}^{\prime \prime}$ satisfy Eq. (62) for $p=2$ or, equivalently, Eq. (52). System II is therefore ideal in backward sense.

$$
M_{a}^{\prime}=\left[\begin{array}{c}
-2 \\
2
\end{array}\right] \quad M_{a}^{\prime \prime}=\left[\begin{array}{l}
-1 \\
-1
\end{array}\right]
$$

System III (arbitrary)

Modifying matrix $N$ of system I to

$$
N=\left[\begin{array}{ll}
0.3 & 1 \\
-1 & 1
\end{array}\right]
$$

is sufficient to lose the above mentioned ideal properties. System III, equal to system I but with $N$ defined by Eq. (74), is therefore an example of arbitrary bilinear system.

\section{ELM Method with Anticausal IOSR}

Consider system II. Its identification via a version of the ELM method based on an anticausal IOSR of order $p_{a}=2$ is exact, as expected thanks to its ideal backward property. The maximum prediction error is around $10^{-13}$, i.e. close to Matlab ${ }^{\circledR}$ working precision. Also, it is worth remarking that the identified bilinear system has the correct dimension $\left(n_{i d}=2\right)$. In contrast, using the version of the ELM method based on the causal IOSR leads to an approximate identification algorithm due to the non-ideal nature of system II in forward sense. For $p_{c}=2$ the identified model is of (wrong) order $n_{i d}=3$ and the prediction accuracy is quite poor (maximum prediction error of $10^{-2}$ ). However, as guaranteed by the convergence theorem, identification accuracy improves as $p_{c}$ is increased. With $p_{c}=4$, for example, the order of the identified system is correctly estimated to be $n_{i d}=2$, and the maximum prediction error is reduced to $10^{-4}$. The anticausal IOSR is therefore a useful complement to the causal IOSR when the ELM method is used, providing more accurate results when the bilinear system is (or at least is closer to being) ideal in backward sense.

\section{IS Method with Causal and Anticausal IOSRs}

Again with reference to system II, the IS identification method can also be applied. For the IS method two IOSRs are needed, and the causal and anticausal IOSRs can serve for the purpose. With system II, which is ideal in backward sense, the causal IOSR is expected to introduce approximation in the identification. In agreement with the convergence theorem, increasing the order of the causal IOSR improves the accuracy of the identified system. While for $p_{c}=p_{a}=2$ the maximum prediction error is around $10^{-2}$, with $p_{c}=6, p_{a}=2$ it gets as low as $10^{-6}$. In both cases, the order of the identified system turns out to be correct $\left(n_{i d}=2\right)$. Note that since none of the presented bilinear systems is ideal in both forward and backward sense, the IS algorithm based on causal and anticausal IOSRs cannot be exact and $p_{c}$ and/or $p_{a}$ must be increased to improve identification.

\section{IS Method with Causal and Mixed-Anticausal IOSRs}

To appreciate how the IS method can benefit from the use of a mixed IOSR, consider system I. Thanks to its forward ideal property, there exist interaction matrices $M_{c}^{\prime}$ and $M_{c}^{\prime \prime}$ such that Eq. (28) is satisfied for $p=2$. Also, the use of the mixed-anticausal IOSR instead of a purely anticausal IOSR relaxes the condition for the existence of $M_{a}^{\prime}$ and $M_{a}^{\prime \prime}$ to the existence of a single $M_{a}^{\prime}$, guaranteed since $A^{-1}$ and $C$ form an observable pair. Identification is therefore expected to be exact when using these IOSRs both of order as low as 2 . This is indeed the case, as confirmed by numerical experiments with $p_{c}=p_{a}=2$, where the maximum prediction error is again of the order of Matlab ${ }^{\circledR}$ working precision. Relying on a mixed IOSR can make the IS identification method exact, in contrast with the IS version based only on pure IOSRs (causal and anticausal).

In order to give more details about the new IS identification method presented in this paper and to remark its effectiveness, a thorough example of its application on an arbitrary bilinear system of the IS method based 
on causal and mixed-anticausal IOSRs is now described. Consider system III and for the sake of generality assume no prior knowledge of its order $n=2$ is available. The a priori guess for the order of the system is, for example, $n=3$. Accordingly, $p_{a}$ is set to be equal to 3 and then $p_{c}$ can be varied to achieve the desired identification accuracy. The following table summarizes the obtained results.

\begin{tabular}{|l|c|c|c|c|c|}
\hline Causal IOSR order $p_{c}$ & 2 & 3 & 4 & 5 & 6 \\
\hline Anticausal IOSR order $p_{a}$ & 3 & 3 & 3 & 3 & 3 \\
\hline Max. prediction error (order of magnitude) & $10^{-1}$ & $10^{-3}$ & $10^{-4}$ & $10^{-5}$ & $10^{-5}$ \\
\hline
\end{tabular}

Note that in the mixed-anticausal IOSR $p_{c}<p_{a}$ is not a consistent choice, since $p_{a}$ must be chosen to be not less than the assumed order of the system and the requirements for the causal interaction matrices are much tougher than those for the (only) anticausal interaction matrix. This observation is well reflected in the previous table. As $p_{c}$ is increased, the prediction accuracy of the identified model improves. Also note that for $p_{c}=6$ the length of the input-output sequences had to be increased to preserve the overdetermined nature of matrix $R$ of Eq. (13). Indeed, as $p_{c}$ and $p_{a}$ are increased, the length of the measured data should increase as well to avoid trivial redundancy of columns of $R$. If the guess on $n$ is improved and the identification algorithm is executed with $p_{a}=2$, the accuracy of results improves accordingly (less numerical noise in the SVDs), as shown in the following table

\begin{tabular}{|l|c|c|c|c|c|}
\hline Causal IOSR order $p_{c}$ & 2 & 3 & 4 & 5 & 6 \\
\hline Anticausal IOSR order $p_{a}$ & 2 & 2 & 2 & 2 & 2 \\
\hline Max. prediction error (order of magnitude) & $10^{-2}$ & $10^{-4}$ & $10^{-5}$ & $10^{-6}$ & $10^{-7}$ \\
\hline
\end{tabular}

For the purpose of showing the key steps of the algorithm, the representative case for $p_{c}=4, p_{a}=3$ is now analyzed more in details. Step 1: Indicating with $Z_{a}$ the superstate history matrix formed with the causal superstate $z_{4, c}(k)$ of Eq. (44) and with $Z_{b}$ the superstate history matrix formed with the mixed-anticausal superstate $z_{4,3, m a}(k)$ of Eq. (67), $Z_{a}$ and $Z_{b}$ have respectively 60 and 193 rows. Step 2: The initial and final time steps for which both IOSRs are valid are $k_{i}=p_{c}=4$ and $k_{f}=l-1-p_{a}=l-4$, giving rise to a matrix $R \in \mathbb{R}^{993 \times 253}$. The singular values of $R$ (normalized over the largest) are shown in Figure 1 . Due to the approximation of the IOSRs, there are no singular values exactly equal to zero and the user has to select the ones that are small enough to suggest some linear dependence of the columns of $R$. This might be a delicate step, but the proposed algorithm proved to be very robust and the user should not be concerned with minimizing at any cost the number $m$ of singular values chosen at this stage. If necessary, the SVD of Step 4 will automatically perform such further reduction. To remark the point, in this specific example all the singular values $10^{4}$ times smaller than the largest are selected (Figure 1), for a total of $m=16$ (instead of selecting $m=2$, which would immediately guarantee $n_{i d}=2$ and lead to similar final results). Step 3: The first 60 entries of each right singular vector $c_{i}, i=1, \ldots, 16$ corresponding to the selected zero singular values are extracted to form column vectors $a_{i}$, and row vectors $h_{i}$ are then computed via the first equality of Eq. (12). Step 4: Matrix $H \in \mathbb{R}^{16 \times 993}$ with $h_{i}$ vectors as rows is constructed and its SVD taken; the graph of normalized singular values of $H$ is shown in Figure 2, from which it is apparent how only two rows of $H$ are linearly independent. Therefore, only two singular values and corresponding right singular vectors are selected and will be used as an estimate of the state history $\{x(k)\}$. The order of the identified bilinear model will then be $n_{i d}=2$. Step 5: Solving the least-squares problems of Eqs. $(17,18)$ leads to the estimate of the following bilinear model matrices (rounded to the $4^{\text {th }}$ significant digit)

$$
\begin{gathered}
A_{i d}=\left[\begin{array}{cc}
-0.8499 & -0.1322 \\
0.3587 & 0.3499
\end{array}\right] \quad B_{i d}=\left[\begin{array}{l}
0.04835 \\
0.08492
\end{array}\right] \\
N_{i d}=\left[\begin{array}{cc}
0.4456 & -0.6513 \\
1.411 & 0.8546
\end{array}\right] \quad C_{i d}=\left[\begin{array}{ll}
20.33 & 11.98
\end{array}\right] \quad D_{i d}=-2.486 \times 10^{-5}
\end{gathered}
$$




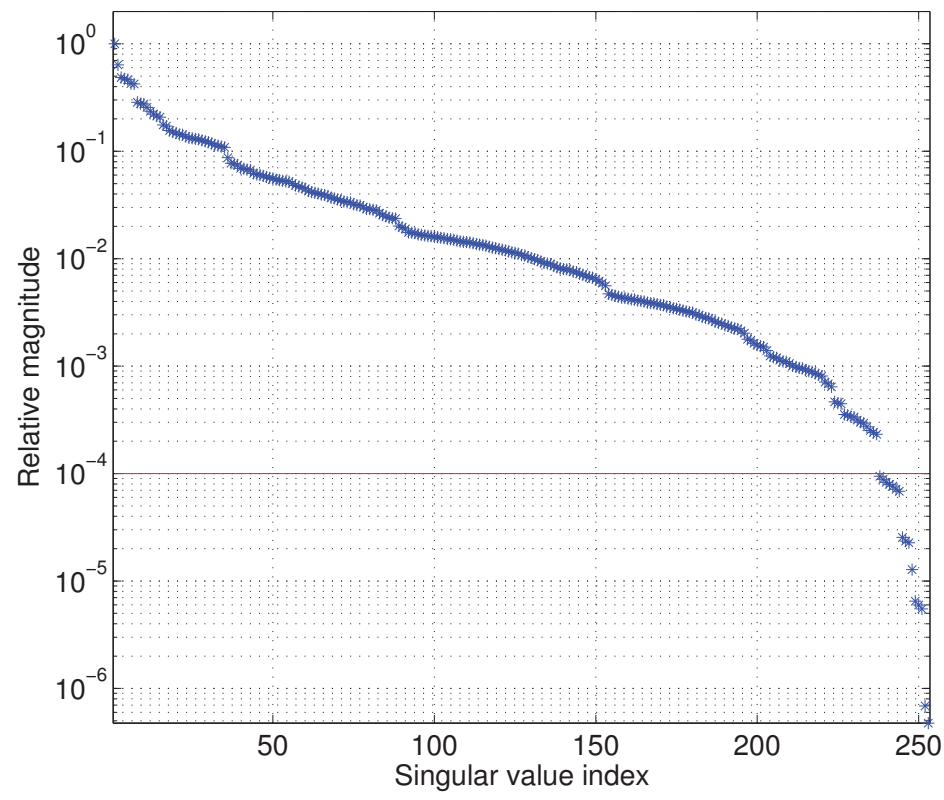

Figure 1: SVD of step 2 of the IS algorithm (to find the intersection of superspaces $Z_{a}$ and $Z_{b}$ ).

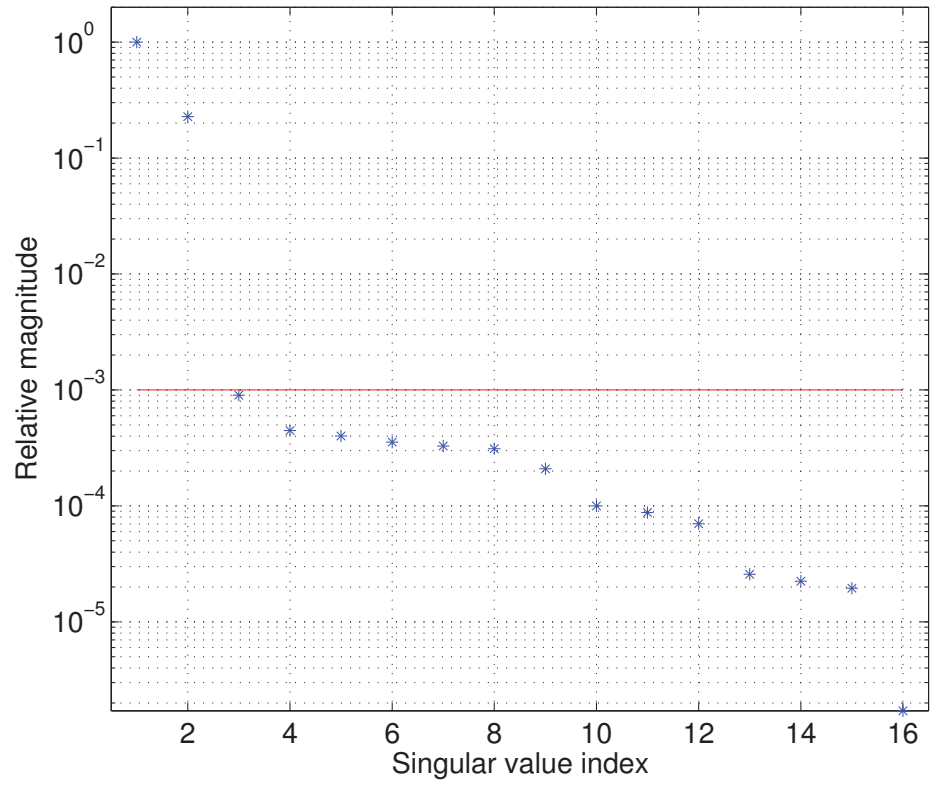

Figure 2: SVD of step 4 of the IS algorithm (to find a minimum basis for the intersection subspace). 

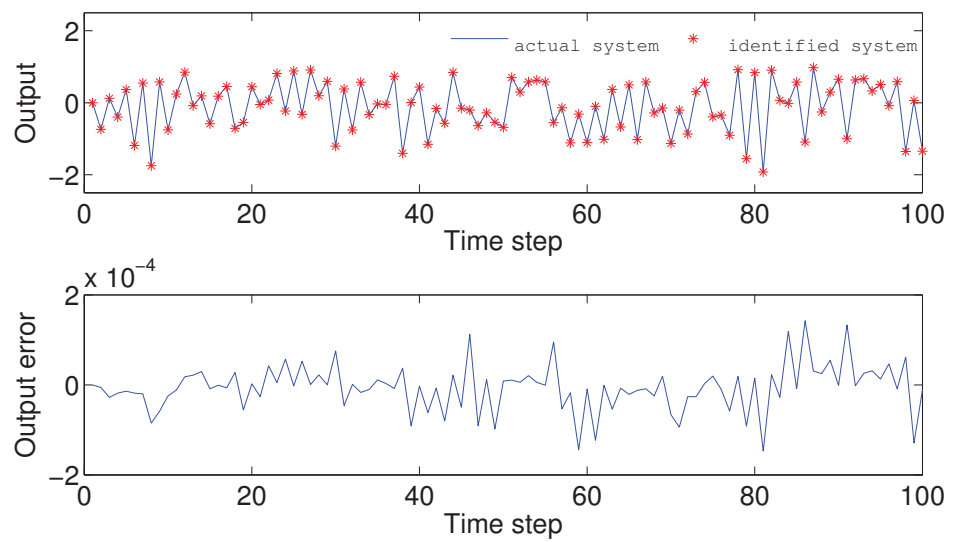

Figure 3: Comparison of output from actual and identified system III.

Figure 3 shows the accuracy of the identified model comparing its predicted output with the output from the actual system III, when both are driven by the same random input sequence, generated independently from the one used to simulate input-output data. As another indicator of the effectiveness of the identification, the following table compares the eigenvalues of the true matrices $A$ and $N$ of the actual system and the eigenvalues of the corresponding identified matrices.

\begin{tabular}{|l|c|c|}
\hline Matrix & Eigenvalue 1 & Eigenvalue 2 \\
\hline$A$ & -0.809016994 & 0.309016994 \\
\hline$A_{i d}$ & -0.809012078 & 0.308957226 \\
\hline Relative error & $0.000608 \%$ & $0.019341 \%$ \\
\hline$N$ & $0.649999999+i 0.936749700$ & $0.649999999-i 0.936749700$ \\
\hline$N_{i d}$ & $0.650096808+i 0.936686271$ & $0.650096808-i 0.936686271$ \\
\hline Relative error & $0.000270 \%$ & $0.000270 \%$ \\
\hline
\end{tabular}

\section{CONCLUSIONS}

This paper has rigorously proven the extension of the interaction matrices to bilinear systems. Interaction matrices were used to derive multiple linear relations between the bilinear system state and input-output data (IOSRs), from which several identification algorithms for bilinear systems were developed. The presented intersection subspace method was shown to be very effective for bilinear system identification. The state of the bilinear system is reconstructed by intersecting vector spaces corresponding to two IOSRs. From the state information, a bilinear state-space model of the system is identified by simple least-squares. A new version of the Equivalent Linear Model approach was also introduced, based on an anticausal IOSR. The benefits of having several IOSRs were highlighted and also shown by numerical examples. In terms of advantages, the approach is applicable to any nonlinear system that can be approximated by the bilinear form given in Eq. (1) as established by Carleman linearization. No specific form of excitation input is required, beyond the standard richness condition for system identification. Also, there is no need to use data from multiple experiments as input-output data from a single experiment is adequate. 
Although we have established the overall theoretical foundation, further research will focus on the practical aspects of the identification algorithms presented in this paper. Accurate identification may require a relatively large value of $p$ which will lead to significant computational burden. Techniques that allow the identification problem to be broken up into a number of smaller problems could be explored. Another practical point is that the convergence of the presented identification algorithms as $p$ increases is guaranteed at the price of keeping the excitation input within a certain bound. This may lead to two potential difficulties. First, the bound is unknown before identification, hence some trial-and-error procedure might be needed. Second, such a bound might turn out to be very small and challenging for practical applications. Despite these potential issues, it is expected that the extension of interaction matrices to bilinear systems will lead to more interesting results on bilinear systems, with the final aim to realize an effective bridge between linear and nonlinear systems in the areas of system identification and control.

\section{REFERENCES}

[1] S. Svoronos, G. Stephanopoulos, and A. Rutherford, "Bilinear Approximation of General Non-Linear Dynamic Systems with Linear Inputs," International Journal of Control, Vol. 31, No. 1, 1980, pp. 109126.

[2] J. T.-H. Lo, "Global Bilinearization of Systems with Control Appearing Linearly," SIAM Journal on Control, Vol. 13, No. 4, 1975, pp. 879-885.

[3] C.-H. Lee and J.-N. Juang, "Nonlinear System Identification - A Continuous-Time Bilinear State Space Approach," Kyle T. Alfriend Astrodynamics Symposium, The American Astronautical Society, Monterey, CA, 2010, AAS10-328.

[4] K. Kowalski and W.-H. Steeb, Nonlinear Dynamical Systems and Carleman Linearization. Singapore: World Scientific, 1991.

[5] J. Minisini, A. Rauh, and E. P. Hofer, "Carleman Linearization for Approximate Solutions of Nonlinear Control Problems: Part 1 - Theory," Advances in Mechanics, Dynamics and Control; Proceedings of the 14th International Workshop on Dynamics and Controls, Moskov, 2007.

[6] A. Rauh, J. Minisini, and E. P. Hofer, "Carleman Linearization for Approximate Solutions of Nonlinear Control Problems: Part 2 - Applications," Advances in Mechanics, Dynamics and Control; Proceedings of the 14th International Workshop on Dynamics and Controls, Moscow, 2007.

[7] M. Q. Phan, Y. Shi, R. Betti, and R. W. Longman, "Discrete-Time Bilinear Representation of Continuous-Time Bilinear State-Space Models," Advances in the Astronautical Sciences, Vol. 143, (to appear).

[8] M. Q. Phan and H. Celik, "A Superspace Method for Discrete-Time Bilinear Model Identification by Interaction Matrices," Advances in the Astronautical Sciences, Vol. 139, 2011, pp. 445-464.

[9] N. Berk Hizir, M. Q. Phan, R. Betti, and R. W. Longman, "Identification of discrete-time bilinear systems through equivalent linear models," Nonlinear Dynamics, Vol. 69, No. 4, 2012, pp. 2065-2078.

[10] W. Favoreel, B. De Moor, and P. Van Overschee, "Subspace identification of bilinear systems subject to white inputs," Automatic Control, IEEE Transactions on, Vol. 44, No. 6, 1999, pp. 1157-1165.

[11] J.-N. Juang, "Generalized Bilinear System Identification," The Journal of the Astronautical Sciences, Vol. 57, No. 1/2, 2009

[12] J.-N. Juang, "Generalized Bilinear System Identification with Coupling Force Variables," Modeling, Simulation and Optimization of Complex Processes (H. G. Bock, X. P. Hoang, R. Rannacher, and J. P. Schlder, eds.), pp. 169-182, Springer Berlin Heidelberg, 2012.

[13] H. Celik and M. Q. Phan, "Identification of Input-Output Maps for Bilinear Discrete-Time State-Space Models," AAS/AIAA Astrodynamics Specialist Conference, Girdwood, AK, 2011, AAS11-426.

[14] P. Van Overschee and B. Moor, Subspace Identification for Linear Systems: Theory, Implementation, Applications. Dordrecht: Kluwer Academic Publishers, 1996.

[15] B. De Moor, M. Moonen, L. Vandenberghe, and J. Vandewalle, "The application of the canonical correlation concept to the identification of linear state space models," Analysis and Optimization of Systems (A. Bensoussan and J. Lions, eds.), Vol. 111 of Lecture Notes in Control and Information Sciences, pp. 1103-1114, Springer Berlin Heidelberg, 1988.

[16] M. Q. Phan, "Interaction Matrices in System Identification and Control," Proceedings of the15th Yale Workshop on Adaptive and Learning Systems, New Haven, CT, 2011.

[17] M. Q. Phan, J.-N. Juang, and R. W. Longman, "Identification of Linear Multivariable Systems by Identification of Observers with Assigned Real Eigenvalues," Journal of the Astronautical Sciences, Vol. 40, No. 2, 1992, pp. 261-27. 
[18] M. Q. Phan, L. G. Horta, J.-N. Juang, and R. W. Longman, "Linear System Identification via an Asymptotically Stable Observer," Journal of Optimization Theory and Applications, Vol. 79, No. 1, 1993, pp. 59-86.

[19] J.-N. Juang, M. Q. Phan, L. G. Horta, and R. W. Longman, "Identification of Observer/Kalman Filter Markov Parameters: Theory and Experiments," Journal of Guidance, Control, and Dynamics, Vol. 16, No. 2, 1993, pp. 320-329.

[20] K. E. Bouazza, M. Boutayeb, and M. Darouach, "State and Output Feedback Stabilization of a Class of Discrete-Time Nonlinear Systems," Proceedings of the 2004 American Control Conference, Vol. 4, 2004, pp. 3023-3028. 